\title{
Über die Krümmung der cyklischen Curven nebst einem Beitrage zur neueren Dreiecksgeometrie.
}

\author{
Nachtrag.
}

\section{Von A. Schwarz in Beneschau.}

Die vorliegende Abhandlung ist eine Frgänzung meines im X. Jahrgange der Monatshefte erschienenen Aufsatzes: "Über die Krümmung der cyklischen Curven etc." Sie ist in zwei Abschnitte getheilt, von denen der erste sich mit der Krümmungsinvolution einer Ellipse beschäftigt. Hierin wird, um nur die hauptsächlichsten Resultate hervorzuheben, von der Gruppe associierter Dreiecke nachgewiesen, dass für alle Tripeldreiecke die Summe der Quadrate ihrer Seiten den gleichen Wert besitzt Ferner wird gezeigt, dass zu jedem Dreiecke zwei andere associjerte gehören, deren Schwerlinien den Seiten des ersteren proportional sind, und umgekehrt; ein Satz, mit Hilfe dessen es in einfacher Weise gelingt, die Dreiecksfläche als Function der Schwerlinien darzustellen. Den Kegelschnitten $K$ und $K^{\prime}$, welche mit der Gruppe associierter Dreiecke in innigem Zusammenhange stehen, werden drei weitere zugesellt, nämlich der imaginäre Kegelschnitt $s_{2} \equiv x_{1}^{2}+x_{2}^{2}+x_{3}^{2}=0$, in Bezug auf welchen die sämmtlichen Tripeldreiecke sich selbst conjugiert sind, und zwei homothetische, mit $K$ und $K^{\prime}$ concentrische Eillipsen, welche von den Höhenschnittpunkten, beziehungsweise den Vittelpunkten der Umkreise sämmtlicher Tripeldreiecke erfüllt werden. Endlich wird auf Grund einer Eigenschaft der Krümmungsinvolution eine eigenthümliche Gleichung für die Kiepert'sche Hyperbel eines. Dreieckes abgeleitet.

Der zweite Abschnitt ist der desmischen Configuration $\left(27_{9}, 81_{3}\right)$ gewidmet, zu welcher eine Curve dritter Ordnung vom Geschlechte: 1 Anlass gibt.

\section{Die Krümmungsinvolution auf einer Ellipse und die Kiepert'sche Hyperbel eines Dreieckes.}

1. Als Krummunginvolution wurde jene cubische Involution auf einem centralen Kegelschnitte $K$ bezeichnet, deren Tripel $r_{1}, r_{2}, r_{3}$ die Eigenschaft besitzen, dass die Krümmungskreise von 
$K$ in diesen Punkten den Träger der Involution an einer und derselben stelle $r$ schneiden. Von dem Dreiecke $r_{1} r_{2} r_{3}$ gilt der Satz, dass die Höhen desselben zugleich Normalen ron $K$ sind, und umgekehrt ist, wie mittelst einer gewissen ein-zweideutigen Verwandtschaft nachgewiesen wurde, jedes mit dieser Eigenschaft behaftete Dreieck ein Tripeldreieck. Indessen ist es möglich, die Giltigkeit dieser Umkehrung in einfacher Weise mittelst des Restsatzes zu begründen, da die dem Dreiecke $r_{1} r_{2} r_{3}$ auferlegte Bedingung sich dahin fassen lässt, dass die drei Punktepaare $r_{1} r_{1}$, $r_{2} r_{2}, r_{3} r_{3}$ beziehungsweise mit $r_{2} r_{3}, r_{3} r_{1}, r_{1} r_{2}$ corresidual sind. Bezeichnet man die Stelle, an welcher der Krümmungskreis in $r_{1}$ dem Kegelschnitte $K$ zum viertennale begegnet, mit $r$, so ist $r r_{1}$ als Residuum von $r_{1} r_{1}$ vermöge des Restsatzes zugleich auch Residuum von $r_{2} r_{3}$, d. h. $r$ wird erhalten, indem man den durch $r_{1} r_{2} r_{3}$ gehenden Kreis mit $K$ zum viertenmale zum Durchschnitte bringt, woraus sich ergibt, dass auch die Krümmungskreise in $r_{2}$ und $r_{3}$ durch $r$ gehen.

2. Eine weitere Eigenschaft der Krümmungsinvolution besteht darin, dass alle Tripeldreiecke $r_{1} r_{2} r_{3}$ einen gemeinschaftlichen Schwerpunkt besitzen, der mit dem Mittelpunkte $c$ von $K$ zusammenfällt. Daraus folgt, dass in der Involution conjugierter Durchmesser von $K$ den Schwerlinien $r_{1} c, r_{2} c, r_{3} c$ beziehungsweise die Durchmesser $D_{1}\left\|r_{2} r_{3}, D_{3}\right\| r_{3} r_{1}, D_{3} \| r_{1} r_{2}$ conjugiert sind. Nun bestehen aber unter der Voraussetzung, dass $K$ mit der Ellipse

$$
K \equiv \frac{x^{2}}{a^{2}}+\frac{y^{2}}{b^{2}}-1=0
$$

identificiert wird, zwischen zwei conjugierten Halbmessern $h, h$, die einen Winkel $\omega$ einschließen, die folgenden Relationen:

$$
\begin{gathered}
h^{2}+h^{\prime 2}=a^{2}+b^{2} \\
h h^{\prime} \sin \omega=a b,
\end{gathered}
$$

welche im vorliegenden Falle die Form annehmen:

$$
\begin{gathered}
\overline{r_{i} c^{2}}+\left(\frac{D_{i}}{2}\right)^{2}=a^{2}+b^{2} \\
\overline{r_{i}} c \cdot \frac{D_{i}}{2} \sin \omega_{i}=a b ;
\end{gathered}
$$

hierin bezeichnet $\omega_{i}$ den Winkel, welchen die durch $r_{i}$ gehende Schwerlinie $t_{i}$ mit der $r_{i}$ gegenüberliegenden Seite $i_{i}$ des Dreieckes $r_{1} r_{2} r_{3}$ bildet.

Zwischen $D_{i}$ und $l_{i}$ besteht ein einfacher Zusammenhang; es ist nämlich bei der Benützung der Parameterdarstellung

$$
x=a \cos \varphi, y=b \sin \varphi
$$


der Parameter eines Endpunktes von $D_{i}$ gleich $\frac{\pi}{2}+\varphi_{i}$, wenn derjenige von $r_{i}$ mit $\varphi_{i}$ bezeichnet wird; daher:

$$
\left(\frac{D_{i}}{2}\right)^{2}=a^{2} \sin ^{2} \varphi_{i}+b^{2} \cos ^{2} \varphi_{i}
$$

Andererseits ist, unter $i, j, k$ den Complex der Indices 1, 2,3 verstanden, wegen

$$
\begin{gathered}
\varphi_{j}=\varphi_{i}+\frac{2 \pi}{3}, \varphi_{i k}=\varphi_{i}+\frac{4 \pi}{3} \\
l_{i}^{2}=a^{2}\left(\cos \varphi_{j}-\cos \varphi_{k}\right)^{2}+b^{2}\left(\sin ^{2} \varphi_{j}-\sin \varphi_{k}\right)^{2}= \\
=4 \sin ^{2} \frac{\varphi_{j}-\varphi_{k}}{2}\left(a^{2} \sin ^{2} \frac{\varphi_{j}+\varphi_{k}}{2}+b^{2} \cos ^{2} \frac{\varphi_{j}+\varphi_{k}}{2}\right)= \\
=3\left(a^{2} \sin ^{2} \varphi_{i}+b^{2} \cos ^{2} \varphi_{i}\right)=3\left(\frac{D_{i}}{2}\right)^{2} \\
l_{i}=\sqrt{3} \frac{D_{i}}{2}, \frac{D_{i}}{2}=\frac{l_{i}}{\sqrt{3}} .
\end{gathered}
$$

Es gehen somit $\left(\alpha^{\prime}\right)$ und $\left(\beta^{\prime}\right)$ in die Gleichungen über

$$
\begin{aligned}
& 4 t_{i}^{2}+3 i_{i}^{2}=9\left(a^{2}+b^{2}\right) \\
& \frac{1}{2} t_{i} l_{i} \sin \omega_{i}=\frac{3 \sqrt{3}}{4} a b,
\end{aligned}
$$

Fon denen die zweite einen neuen Beweis dafür erbringt, dass die Tripeldreiecke eine constante Fläche haben, während $\left(\alpha^{\prime \prime}\right)$ die beiden folgenden Sätze liefert:

Für alle Tripeldreiecke ist die Summe der Quadrate ihrer Seiten constant, nämlich $=\frac{9}{2}\left(a^{2}+b^{2}\right)$.

Die Summe der Quadrate der durch die Ecken eines Tripeldreieckes gehenden Halbmesser $\overline{r_{1} c}, \overline{r_{2} c}, \overline{r_{3} c}$ von $K$ besitzt zugleich mit der Summe der Quadrate der diesen Halbmessern conjugierten Halbmesser den constanten Wert $\frac{3}{2}\left(a^{2}+b^{2}\right)$.

In der That folgt durch Addition der beiden Gleichungen

$$
\begin{aligned}
& l_{1}^{2}=t_{3}^{2}+\frac{1}{4} l_{3}^{2}-t_{3} l_{3} \cos \omega_{3} \\
& l_{2}^{2}=t_{3}^{2}+\frac{1}{4} l_{3}^{2}-t_{3} l_{3} \cos \omega_{3}
\end{aligned}
$$


die Relation

$$
l_{1}^{2}+l_{2}^{2}=2 t_{3}^{2}+\frac{1}{2} l_{3}^{2}
$$

oder

$$
l_{1}+l_{2}^{2}+l_{3}^{2}=\frac{1}{2}\left(4 t_{3}^{2}+3 l_{3}^{2}\right)
$$

welche in Verbindung mit ( $\left.\alpha^{\prime \prime}\right)$

$$
l_{1}^{2}+l_{2}^{2}+l_{3}^{2}=\frac{9}{2}\left(a^{2}+b^{2}\right)
$$

ergibt. Aus $(\gamma)$ folgt alsdann:

$$
\left(\frac{D_{1}}{2}\right)^{2}+\left(\frac{D_{2}}{2}\right)^{2}+\left(\frac{D_{3}}{2}\right)^{2}=\frac{3}{2}\left(a^{2}+b^{2}\right)
$$

und vermöge $\left(a^{\prime}\right)$

$$
\overline{r_{1} c^{2}}+\overline{r_{2} c^{2}}+\overline{r_{3} c^{2}}=\frac{3}{2}\left(a^{2}+b^{2}\right) .
$$

Die Gleichungen $\left(\Omega^{\prime \prime}\right)$ und ( $(\hat{)})$ ermöglichen es, die Achsen der Ellipse $K$ zu berechnen, für welche ein vorgelegtes Dreieck $r_{1} r_{2} r_{3}$ ein Tripeldreieck der zugehörigen Krümmungsinvolution darstellt.

Die in diesem \& erhaltenen Resultate können im folgenden Satze zusammengefasst werden: Projiciert man die Krümmungsinvolution auf $K$ aus dem Mittelpunkte $c$ des letzteren, so ergibt sich eine eubische Involution der Halbmesser, welche ähnliche Eigenschaften aufweist, wie die Involution corjugierter Halbmesser, indem jedes Tripel $h_{1}, h_{2}, h_{3}$ derselben den Gleichungen genügt:

$$
h_{1}^{2}+h_{2}^{2}+h_{3}^{2}=\frac{3}{2}\left(a^{2}+b^{2}\right)
$$

$$
h_{1} h_{2} \sin \left(h_{1} h_{2}\right) \sin +h_{2} h_{3} \sin \left(h_{2} h_{3}\right)+h_{3} h_{1} \sin \left(h_{3} h_{1}\right)=\frac{3 \sqrt{3}}{2} a b,
$$

wenn unter $\left(h_{1} h_{2}\right)$ der Winkel verstanden wird, den die Halbmesser $h_{1}, h_{2}$ mit einander einschließen.

$\mathrm{Da}$ die Krümmungsinvolution $\lambda^{3}=$ const mit jedem Tripel $r_{1}, r_{2}, r_{3}$ zugleich auch das Tripel der diametral gegenüberliegenden Punkte $r_{1}^{\prime}, r_{2}^{\prime}, r_{3}^{\prime}$ enthält, deren Parameter sich von denen der entsprechenden nicht accentuierten nur durch das Vorzeichen unterscheiden, so wird die eubische Involution der Halbmesser von selbst zu einer solchen der Durchmesser.

Überdies hat sie die Eigenschaft, sich selbst conjugiert zu sein, denn die zu $\overline{r_{1} c}, \overline{r_{2} c}, \overline{r_{3} c}$ conjugierten Durchmesser enthalten 
jene drei Punkte, deren Parameter $\lambda$ aus denen von $r_{1}, r_{2}, r_{3}$ durch Multiplication mit $i$ gewonnen werden.

3. Die Formel ( $\%$ ) des $\S .2$ gestattet auch die folgende Schreibweise:

$$
\begin{aligned}
l_{i}^{2} & =3\left(\frac{D_{i}}{2}\right)^{2}=3\left(a^{2} \sin ^{2} \varphi_{i}+b^{2} \cos ^{2} \varphi_{i}\right)= \\
& =3\left[a^{2} \cos ^{2}\left( \pm \frac{\pi}{2}-\varphi_{i}\right)+b^{2} \sin ^{2}\left( \pm \frac{\pi}{2}-\varphi_{i}\right)\right],
\end{aligned}
$$

durch we'che das Dreieck $r_{1} r_{2} r_{3}$ zu zwei anderen Tripeldreiecken in Beziehung gisetzt wird, deren Ecken, den beiden Vorzeichen entsprechend, die Parameter

$$
\psi_{i}= \pm \frac{\pi}{2}-\varphi_{i} \quad(i=1,2,3)
$$

besitzen. Um einen bestimmten Fall vor Augen zu haben, möge zunächst nur das obere Vorzeichen berücksichtigt werden. Bezeichnet. man alsdann die Seiten und Schwerlinien des Tripeldreieckes $\left(+\frac{\pi}{2}-\varphi_{i}\right)(i=1,2,3)$ mit $i_{1}^{\prime}, l_{2}^{\prime}, l_{3}^{\prime}$, bezw. $t_{1}^{\prime}, t_{2}^{\prime}, t_{3}^{\prime}$, so ist klar, dass der Ausdruck in der eckigen Klammer den Wert $\left(\frac{2}{3} t_{i}^{\prime}\right)^{2}$ besitzt. Es ist daher

$$
l_{i}=\frac{2}{\sqrt{3}} t_{i}^{\prime}
$$

Andererseits lehrt die Gleichung

$$
p_{i}+\psi_{i}=+\frac{\pi}{2}
$$

wegen ihres symmetrischen Baues in den Parametern $\varphi_{i}$ und $\psi_{i}$, dass die Beziehung zwischen den beiden in Betracht kommenden Tripeldreiecken eine wechselseitige ist, weshalb auch die Gleichung:

$$
l_{i}^{\prime}=\frac{2}{\sqrt{3}} t_{i}
$$

Geltung hat. Mit anderen Worten: Die beiden Tripeldreiecke sind von der: Beschaffenheit, dass die Seiten des einen den Schwerlinien des anderen proportional sind, und umgekehrt. Dabei versteht es sich von selbst, dass dieser Satz auch dann aufrecht bleibt, wenn an Stelle des Dreieckes $\left(+\frac{\pi}{2}-\varphi_{i}\right)$ das durch $\left(-\frac{\pi}{2}-\varphi_{i}\right)$ charakterisierte gesetzt wird. 
Was die Lage der beiden letztgenannten Dreiecke anlangt, so ergibt sie sich am einfachsten durch Rückkehr zu dem Parameter $\lambda$, da für $\lambda_{i}=e^{i \varphi_{i}}, \mu_{i}=e^{i \psi_{i}}$ aus $\varphi_{i}+\psi_{i}= \pm \frac{\pi}{2}$

$$
\lambda_{i} \mu_{i}= \pm i
$$

hervorgeht; d. h. die drei Strahlen $\overline{\lambda_{i}} \bar{\mu}_{i}(i=1,2,3)$ sind, den beiden Vorzeichen entsprechend, bezw. den Strahlen $\overline{1(+i)} \equiv \overline{(a, o)(a, b)}$ und $\overline{1(-i)} \equiv \overline{(a, o)(o,-b)}$ parallel.

Mit Hilfe der Gleichung $l_{i}^{\prime}=\frac{2}{\sqrt{3}} t_{i}$ kann man die Fläche $f$ des Dreieckes $r_{1} r_{2} r_{3}$, welches ein ganz beliebiges Dreieck ist, durch seine Schwerlinien ausdrücken. Aus der Constanz des Flächeninhaltes der Tripeldreiecke folgt nämlich, dass die Fläche des Dreieckes mit den Seiten $l_{1}^{\prime}, l_{2}^{\prime}, l_{3}^{\prime}$ ebenfalls den Wert $f$ besitzt, so dass die Gleichung besteht:

$$
\begin{gathered}
f=\sqrt{L^{\prime}\left(L^{\prime}-l_{1}^{\prime}\right)\left(L^{\prime}-l_{2}^{\prime}\right)\left(L^{\prime}-\bar{l}_{3}^{\prime}\right)} \\
l_{1}^{\prime}+l_{2}^{\prime}+l_{3}^{\prime}=2 L^{\prime} .
\end{gathered}
$$

Setzt man

so ist

$$
t_{1}+t_{2}+t_{3}=2 T
$$

daher

$$
L^{\prime}=\frac{2}{\sqrt{3}} T, \quad L^{\prime}-l_{i}^{\prime}=\frac{2}{\sqrt{3}}\left(T-t_{i}\right)
$$

$$
f=\frac{4}{3} \sqrt{T\left(T-t_{1}\right)\left(T-t_{2}\right)\left(T-t_{3}\right)} .
$$

4. Von den Affinitäten, welche den Kegelschnitt $K$ in sich überführen, wurde nachgewiesen, dass sie zugleich auch Transformationen der Krümmungsinvolation, und infolgedessen der Gruppe associierter Dreiecke, in sich sind. Unter denselben nehmen die durch

$$
\lambda^{\prime}=c \lambda \text { oder } \varphi^{\prime}=\varphi+k
$$

charakterisierten eine ausgezeichnete Stellung ein, indem sie die unendlich fernen Punkte von $K$ einzeln invariant lassen. Die Gesammtheit dieser letzteren Aftinitäten bildet eine continuierliche eingliedrige Gruppe $G$, welche zufolge der für die Multiplication, beziehungsweise Addition giltigen Operationsgesetze — des associativen und commutativen - mit ihrer Parametergruppe identisch und als Abel'sche Gruppe zu bezeichnen ist, da irgend zwei Transformationen derselben vertauschbar sind.

Was die Darstellung der Gruppe $G$ in trimetrischen Coordinaten anlangt, so wird dieselbe durch das Formelsystem 


$$
\begin{aligned}
& y_{1}+y_{2}+y_{3}=A\left(x_{1}+x_{2}+x_{3}\right) \\
& y_{1}+\rho y_{2}+p^{2} y_{3}=B\left(x_{1}+p x_{2}+\rho^{2} x_{3}\right) \\
& y_{1}+\rho^{2} y_{2}+p y_{3}=C\left(x_{1}+\rho^{2} x_{2}+\rho x_{3}\right)
\end{aligned}
$$

geleistet, woferne nur die Constanten $A, B, C$ derart bestimmt werden, dass durch die angeführte Collineation $K \equiv x_{1} x_{2}+x_{2} x_{3}+x_{3} x_{1}=0$ in sich übergeführt wird; eine Forderung, welcher die Bedingungsgleichung:

$$
A^{2}=B C
$$

genügt. Durch Auflösung des Systems (9) nach $y_{1}, y_{2}, y_{3}$ ergibt sich

$y_{1}=\alpha\left(x_{1}+x_{2}+x_{3}\right)+\beta\left(x_{1}+p x_{2}+p^{2} x_{3}\right)+\gamma\left(x_{1}+p^{2} x_{2}+p x_{3}\right)$ $y_{2}=\alpha\left(\infty_{1}+x_{2}+x_{3}\right)+\beta p^{2}\left(x_{1}+p x_{2}+p^{2} x_{3}\right)+\gamma p\left(x_{1}+p^{2} x_{2}+p x_{3}\right)$ $y_{3}=\alpha\left(x_{1}+x_{2}+x_{3}\right)+\beta \rho\left(x_{1}+\rho x_{2}+\rho^{2} x_{3}\right)+\gamma \rho^{2}\left(x_{1}+\rho^{2} x_{2}+\rho x_{3}\right)$,

oder

$y_{1}=(\alpha+\beta+\gamma) x_{1}+\left(\alpha+\beta p+\gamma p^{2}\right) x_{2}+\left(\alpha+\beta p^{2}+\gamma p\right) x_{3}$ $y_{2}=\left(\alpha+\beta p^{2}+\gamma p\right) x_{1}+(\alpha+\beta+\gamma) x_{2}+\left(\alpha+\beta p+\gamma p^{2}\right) x_{3}$ $y_{3}=\left(\alpha+\beta \rho+\gamma \rho^{2}\right) x_{1}+\left(\alpha+\beta \beta^{2}+\gamma \rho\right) x_{2}+(\alpha+\beta+\gamma) x_{3}$,

so dass die in Frage stehenden Affinitäten durch das Schema

$$
S=\left(\begin{array}{lll}
a_{11} & a_{12} & a_{13} \\
a_{13} & a_{11} & a_{12} \\
a_{12} & a_{13} & a_{11}
\end{array}\right)
$$

im Vereine mit der $A^{2}-B C=0$ äquivalenten Bedingungsgleichung:

$$
a_{11} a_{12}+a_{13} a_{12}+a_{11} a_{13}=0
$$

charakterisiert sind. Es sind demnach die in $\tilde{s}$ auftretenden Coefficienten $a_{11}, a_{12}, a_{13}$ mit den Coordinaten eines Punktes von $K$ zu identificieren, was durch die Annahme

erzielt wird.

$$
\sigma a_{11}=-x, \sigma a_{12}=1+x, \sigma a_{13}=x(1+x)
$$

Hinsichtlich des Schemas $S$ soll noch bemerkt werden, dass dasselbe, abgesehen von der Hauptdiagonale von links oben nach rechts unten, die Eigenschaft der magischen Quadrate aufweist, indem die Summe der Elemente in jeder Horizontal- und jeder Verticalreihe, ebenso wie in der Nebendiagonale von rechts oben nach links unten die gleiche ist.

5. Für die Transformationen $S$ der Gruppe $G$ ist die Fläche eine Invariante. Denn für eine affine Verwandtschaft ist das Verhältnis zwischen den Flächen von Bild und. Original einer Constanten 
gleich, welche im vorliegenden Falle den Wert 1 besitzt, weil durch jede Transformation $S$ das Innere der Ellipse $K$ in sich äberführt wird.

Übrigens ergibt sich dies auch aus der folgenden Darstellung von $S$ :

$$
\begin{aligned}
& \frac{2 \xi}{a}=\left(c+\frac{1}{c}\right) \frac{x}{a}+\left(c-\frac{1}{c}\right) \frac{i y}{b} \\
& \frac{2 i \eta}{b}=\left(c-\frac{1}{c}\right) \frac{x}{a}+\left(c+\frac{1}{c}\right) \frac{i y}{b},
\end{aligned}
$$

welche der für eine flächentrene oder äquivalente Abbildung giltigen Bedingungsgleichung

genügt.

$$
\frac{\partial \xi}{\partial x} \frac{\partial \eta}{\partial y}-\frac{\partial \xi \partial \eta}{\partial y} \frac{\partial \eta}{\partial x}= \pm 1
$$

Als unmittelbare Anwendung davon erscheint der Satz von der Constanz des Flächeninhaltes der Tripeldreiecke, da irgend zwei derselben durch eine Transformation der Gruppe $G$ in einander verwandelbar sind.

$\mathrm{Zu}$ einer zweiten Anwendung wird man durch die Frage nach den Bahncurven dieser Gruppe geführt. Unter einer Bahncurve versteht man bekanntlich die Linie, welche ein Punkt $x$ bei Variation des Parameters einer continuierlichen eingliedrigen Gruppe beschreibt. Lässt man $x$ mit einem Punkte von $K$ coincidieren, so ergibt sich als zugehörige Bahneurve der Kegelschnitt $K$ selbst. Andererseits ist klar, dass das Büschel homothetischer Kegelschnitte

$$
\frac{x^{2}}{a^{2}}+\frac{y^{2}}{b^{2}}=k
$$

sich bei Anwendung irgend einer Operation von $G$ reproduciert, und zwar wegen des Invariantencharakters des Flächeninhaltes so, dass jede einzelne Linie desselben in sich ubergeht. Damit ist in diesem Büschel das System der Bahncurven gefunden.

$\mathrm{Zu}$ ihrer Bestimmung kann als vermittelnder Gedanke auch eine Cayley'sche Maßbestimmung dienen, deren Fundamentalkegelschnitt mit $K$ zusammenfällt, da unter dieser Voraussetzung $\frac{x^{2}}{a^{2}}+\frac{y^{2}}{b^{2}}=k$ ein Büschel concentrischer Kreise darstellt, von denen jeder eine Bahncurve von $G$ reprösentiert, weil die Operationen dieser Gruppe in Drehungen um den gemeinschaftlichen Mittelpunkt $(0,0)$ bestehen. Bei diesem Nicht-Euklidischen Messungsverfahren sind sämmtliche Bahncurven als confocal anzusehen.

Unter den invarianten Curven nehmen zwei ein besonderes Interesse in Anspruch, nämlich erstens der Involutionskegelschnitt $K^{\prime}$ der Krümmungsinvolution, und zweitens jener Kegelschnitt $K^{\prime \prime}$, 
in Bezug auf welchen nach einem bekannten Satze, von dem Herr $G$. Kohn eine Verallgemeinerung angegeben hat ${ }^{1}$, alle Tripeldreiecke sich selbst conjugiert sind. Zur Feststellung ihrer Gleichungen reicht die Betrachtung eines Tripeldreieckes aus, dem ein Scheitel von $K$, etwa der Punkt $(a, 0)$, als Ecke angehört. Seine Gegenseite $x=-\frac{a}{2}$ ist nämlich eine Tangente von $K^{\prime}$, deren Berührungspunkt natürlich in die Stelle $\left(-\frac{a}{2}, o\right)$ fällt, weshalb

$$
K^{\prime} \equiv \frac{x^{2}}{a^{2}}+\frac{y^{2}}{b^{2}}-\frac{1}{4}=0 \text {. }
$$

Ferner ist $x=-\frac{a}{2}$ die Polare von $(a, 0)$ in Bezug auf

$$
K^{\prime \prime}=\frac{x^{2}}{a^{2}}+\frac{y^{2}}{b^{2}}-k=0
$$

woraus

$$
k=\frac{-1}{2}
$$

hervorgeht. Man kann sich auch a posteriori leicht überzeugen, dass der Kegelschnitt

$$
\frac{x^{2}}{a^{2}}+\frac{y^{2}}{b^{2}}+\frac{1}{2}=0
$$

thatsächlich mit $K^{\prime \prime}$ identisch ist. Die Gleichung der einem Punkte $(a \cos \varphi, b \sin \varphi)$ zugehörigen Polare von $\frac{x^{2}}{a^{2}}+\frac{y^{2}}{b^{2}}+\frac{1}{4}=0$ lautet nämlich

$$
\frac{x \cos \varphi}{a}+\frac{y \sin \varphi}{b}=-\frac{1}{2}
$$

und wegen

$\cos \left(\varphi+\frac{2 k \pi}{3}\right) \cos \varphi+\sin \left(\varphi+\frac{2 k \pi}{3}\right) \sin \varphi=\cos \frac{2 k \pi}{3}=-\frac{1}{2}(k=1,2)$

genügen ihr thatsächlich die Coordinaten der den Parameterwerten $\varphi+\frac{2 \pi}{3}$ and $\varphi+\frac{4 \pi}{3}$ entsprechenden Punkte von $K$.

Es erübrigt noch, die Gleichung von $K^{\prime \prime}$ in homogenen Coordinaten anzugeben. $\Lambda$ ls einer Bahncurve von $G$ angehörig ist dieselbe nothwendig von der Form

$$
\left(x_{1}+x_{2}+x_{3}\right)^{2}-k\left(x_{1} x_{2}+x_{2} x_{3}+x_{3} x_{1}\right)=0,
$$

1) Vgl. Wiener Sitzungsbericht C. 6-19. G. Kohn. Über einige projective Eigenschaften der Poncelet'sehen Polygone. 
wobei $k$ so zu bestimmen ist, dass der Punkt $(1,0,0)$ die Grade $x_{1}=0$ zur Polaren hat. Aus dieser Bedingung folgt

so dass

$$
k=2
$$

$$
K^{\prime \prime} \equiv x_{1}^{2}+x_{2}^{2}+x_{3}^{2}=0
$$

ist; ein Resultat, welches mit der Theorie der algebraischen Gleichung dritten Grades im Zusammenhange steht, indem es die geometrische Bedeutung der gleich Null gesetzten zweiten Potenzsumme der Wurzeln einer solchen Gleichung liefert.

6. Im vorhergehenden §. wurde nachgewiesen, dass die Substitutionen der Gruppe $G$ Automorphien der ambigen quadratischen Form $\frac{x^{2}}{a^{2}}+\frac{y^{2}}{b^{2}}$ sind. Geometrisch findet diese Thatsache ihren Ausdruck in einem Satze, welcher aus der geometrischen Bedeutung von $K \equiv \frac{x^{2}}{a^{2}}+\frac{y^{2}}{b^{2}}-1$ hervorgeht.

Man findet nämlich für den Abstand $p$ eine Punktes $(\xi, \eta)$ von seiner Polaren $P$ in Bezug auf den Kegelschnitt $K=0$ den Wert

$$
p=\frac{K(\xi, \eta)}{\sqrt{\frac{\xi^{2}}{a^{4}}+\frac{r_{i}^{2}}{b^{2}}}}= \pm p^{\prime} K(\xi, \eta)
$$

wenn $x^{\prime}$ die Länge des vom Anfangspunkte $o$ der Coordinaten auf $P$ gefällten Perpendikels bezeichnet. Es ist demnach

$$
K(\xi, \eta)=\varepsilon \frac{p}{p^{\prime}},
$$

wo $\varepsilon= \pm 1$ ist, je nachdem $(\xi, \eta)$ anßerhalb oder innerhalb der Ellipse $\bar{K}$ liegt.

Dies Resultat ist auch dann noch richtig, wenn unter $K(x, y)$ die um die Einheit verminderte binäre Form von der $n^{\text {ten }}$ Dimension $K_{n}(x, y) \equiv f_{n}(x, y)-1 \equiv a_{n, 0} x^{n}+a_{n-1,1} x^{n-1} y+\cdots+a_{0, n} y^{n}-1$ verstanden wird. Unter dieser Voraussetzung ist nämlich die grade Polare $P$ des Punktes $(\xi, \eta)$ in Bezug auf $K_{n}(x, y)=0$ gegeben dureh die Gleichung

$$
\frac{1}{n}\left(\frac{\partial f_{n}}{\partial \xi} x+\frac{\partial f_{n}}{\partial \eta} y\right)=1
$$

aus welcher mit Benutzung von

$$
\frac{1}{n}\left(\xi \frac{\partial f_{n}}{\partial \dot{\xi}}+\eta \frac{\partial f_{n}}{\partial \eta}\right)=f_{n}(\xi, \eta)
$$

wiederum die Gleichung ( $\iota$ gewonnen wird. 
Für $n=2$ und $K_{2} \equiv \frac{x^{2}+y^{2}}{r^{2}}-1$, d. i. für den Kreis, fallen $p$ und $p^{\prime}$ in den Strahl $m o$, wenn $m \equiv(\xi, n)$ ist. Ist alsdann $n$ der Berührangspunkt einer der beiren von $m$ an den Kreis $K_{2}=0$ gehenden Tangenten und $q$ der Fußpunkt des von $n$ auf $o m$ gefällten Perpendikels, so folgt aus der Áhnlichkeit der Dreiecke $m n q$ und $o n m$ wenn $\overline{m n}=t, \overline{n q}=l$ gesetzt wird:

$$
\begin{gathered}
\frac{l}{p^{\prime}}=\frac{t}{r}, l=\sqrt{p p^{\prime}}, \sqrt{\frac{p}{p^{\prime}}}=\frac{t}{r} \\
K(\xi, \eta)=\varepsilon \frac{t^{2}}{r^{2}} \\
\xi^{2}+\eta^{2}-r^{2}=\Xi t^{2} .
\end{gathered}
$$

Für den Kreis ist demnach die Gleichung $K(\xi, \eta)=\varepsilon \frac{p}{p^{\prime}}$, identisch mit dem bekannten Satze, dass $\xi^{2}+\eta^{2}-r^{2}$ die Potenz des Punktes $\left(\xi, r_{1}\right)$ in Bezug auf den Kreis $K=0$ darstellt. Ebenso ist die Eigenschaft des Kreises $x^{2}+y^{2}=R^{2}$, der geometrische Ort solcher Punkte zu sein, die in Bezug auf $x^{2}+y^{2}=r^{2}$ gleiche Potenzen von bestimmter Größ̉e haben, als Specialfall in dem Theoreme enthalten, wonach für alle Punkte der Ellipse $\frac{x^{2}}{a^{2}}+\frac{y^{2}}{b^{2}}=k$ der Quotient $\frac{p}{p^{\prime}}$ eine Invariante ist.

Der Bruch $\frac{p}{p^{\prime}}$ auf der linken Seite der Gleichnng (l) ist, vom Vorzeichen abgesehen, das in Bezug auf die Fundamentalpunkte $o, m$ gebildete Theilverhältnis des Punktes $s$, in welchem der Strabl o $m$ der Polare $P$ begegnet. Daher bestehen unter $u_{\infty}$ den unendlich fernen Punkt von $o m$ verstanden, die Gleichungen

$$
\begin{aligned}
& -\varepsilon \frac{p}{p^{\prime}}=\left(\text { oms } u_{\infty}\right) \\
& K\left(\xi, r_{1}\right)=-\left(o m s u_{\infty}\right) ;
\end{aligned}
$$

mit andern Worten: Das Resultat der Substitution der Coordinaten eines Punktes $m$ in die linke Seite der Gleichung $K=0$ ist gleich dem negativ genommenen Doppelverhältnisse ( $m s u_{\infty}$ ), wenn mit $s, u_{\infty}$ die Punkte bezeichnet werden, in denen $o m$ die zu $m$ und $o$ gehörigen Polaren von $K=0$ schneidet.

Und nun ist es, mit Rücksicht auf die letzte Gleichung, geometrisch evident, dass die binäre quadratische Form $\frac{x^{2}}{a^{2}}+\frac{y^{2}}{b^{2}}$ bei Anwendung einer jeden Substitution der Gruppe $G$ invariant bleibt.

7. Der Umstand, dass die Form $\frac{x^{2}}{a^{2}}+\frac{y^{2}}{b^{2}}$ eine Invariante von $G$ ist, legt die Vermuthung nahe, dass die Eigenschaften der Kr'üm- 
mungsinvolution sich in besonders einfacher Weise werden beschreiben lassen, wenn an Stelle der gewöhnlichen Maßbestimmung eine pseudometrische zugrunde gelegt wird, bei welcher die absoluten Kreispunkte durch die auf der unendlich fernen Graden gelegenen Verschwindungspunkte der positiven Form

$$
f(x, y) \equiv \frac{x^{2}}{a^{2}}+\frac{y^{2}}{b^{2}}
$$

ersetzt werden.

Für die Distanz eines Punktes $(x, y)$ vom Anfangspunkte der Coordinaten, und allgemeiner für die Entfernung zweier Punkte $(x, y),\left(x^{\prime} y^{\prime}\right)$ gelten unter dieser Voraussetzung die folgenden Definitionen :

$$
d=\sqrt{f\left(x, y^{\prime}\right)} \text { bezw. }=\sqrt{f\left(x-\overline{x^{\prime}}, \bar{y}-y^{\prime}\right)},
$$

während zwei Strahlen, die den Anfangspunkt mit den Punkten $(x, y)$ und $\left(x^{\prime}, y^{\prime}\right)$ verbinden, einen Winkel

$$
\begin{gathered}
\Theta=\arccos \frac{\frac{1}{2}\left(x^{\prime} \frac{\partial f}{\partial x}+y \frac{\partial f}{\partial y}\right)}{\sqrt{f(x, y)} \sqrt{f\left(x^{\prime}, y^{\prime}\right)}}=\arccos \frac{\frac{1}{2}\left(x \frac{\partial f}{\partial x^{\prime}}+y \frac{\partial f}{\partial y^{\prime}}\right)}{\sqrt{f(x, y)} \sqrt{f\left(x^{\prime}, y^{\prime}\right)}}= \\
=\arccos \frac{\frac{x x^{\prime}}{a^{2}}+\frac{y y^{\prime}}{b^{2}}}{\sqrt{\frac{x^{2}}{a^{2}}+\frac{y^{2}}{b^{2}}} \sqrt{\frac{x^{\prime 2}}{a^{2}}+\frac{y^{\prime 2}}{b^{2}}}}
\end{gathered}
$$

einschließen. Durch Nullsetzen der Linienfactoren

$$
\lambda \equiv \frac{x}{a}+\frac{i y}{b}, \mu \equiv \frac{x}{a}-\frac{i y}{b},
$$

in welche die Form $f$ sich zerlegen lässt, erhält man die Minimallinien der Maßbestimmung, deren Minimalcoordinaten also mit $\lambda, \mu$ identisch sind.

Auf der Peripherie der Ellipse $K$, welche nun als Einheitskreis $f \equiv \lambda \mu=1$ anzuseben ist, sind $\lambda$ und $\mu$ reciprok, wie dies auch aus der Parameterdarstellang:

ersichtlich ist.

$$
\frac{2 x}{a}=\lambda+\frac{1}{\lambda}, \frac{2 i y}{b}=\lambda-\frac{1}{\lambda}
$$

Um die Eigenschaften der Krümmungsinvolution in der neuen Maßbestimmung $\mathrm{zu}$ beschreiben, ist es angezeigt, die pseudometrische Entfernung $d$ zweier Punkte $m_{1}, m_{2}$ von $K$, sowie den pseudometrischen Winkel, welchen die Radien o $m_{1}$, o $m_{2}$ einschließen, durch die zu $m_{1}, m_{2}$ gehörigen Minimalcoordinaten $\lambda_{1}, \lambda_{2}$, 
bezichungsweise die Winkelcoordinaten $\varphi_{1}, \varphi_{2}$ auszudrücken. Mittelst der Definitionsgleichungen $(x),(\lambda)$ findet man:

$$
\begin{gathered}
d=i \frac{\lambda_{1}-\lambda_{2}}{\sqrt{\lambda_{1} \lambda_{2}}}=i\left(\sqrt{\frac{\lambda_{1}}{\lambda_{2}}}-\sqrt{\frac{\lambda_{2}}{\lambda_{1}}}\right)=2 \sin \frac{\varphi_{1}-\varphi_{2}}{2} \\
\theta=\arccos \frac{1}{2}\left(\frac{\lambda_{1}}{\lambda_{2}}+\frac{\lambda_{2}}{\lambda_{1}}\right)=\varphi_{1}-\varphi_{2},
\end{gathered}
$$

wozu noch die Formel für das Bogenelement hinzukommt:

$$
d s=i \frac{d \lambda}{\lambda}=d \theta
$$

Diese Gleichungen können ohne alle Rechnung erhalten werden, wenn man sich der Abbildung

$$
\dot{\varepsilon}=\frac{x}{a}, \pi=\frac{y}{b}
$$

bedient, durch welche die Grundpunkte der Cayley'schen Maßbestimmung in der $x y$ Ebene in die absoluten Kreispunkte der $\xi_{\eta}$ Ebene übergehen. Damit wird nämlich erreicht, dass die pseudometrische Entfernung zweier Punkte und der pseudometrische Winkel zweier Strahlen der Originalebene gleich werden der Entfernung der homologen Punkte, beziehungsweise dem Winkel der entsprechenden Strahlen in der Bildebene, wobei in der letzteren die gewöhnliche Maßbestimmung anzuwenden ist.

Da die Tripeldreiecke der Krümmungsinvolution sich in dem Systeme der dem Einheitskreise $\xi^{2}+r^{2}=1$ eingeschriebenen gleichseitigen Dreiecke abbilden, so sind dieselben in der vorliegenden Pseudometrik als gleichseitig anzusehen, und zwar ist die Seitenlänge $=2 \sin \frac{\pi}{3}=\sqrt{\mathbf{3}}$. Ferner erscheint eine jede Seite eines Tripeldreieckes, vom Anfangspunkte $o$ aus gesehen, unter einem Winkel von $120^{\circ}$, wie dies auch die Formel für $\cos \theta$ ergibt, wenn hierin für $\lambda_{2}$ der Wert $p \lambda_{1}$ eingeführt wird:

$$
\begin{aligned}
\cos \theta=\frac{1}{2}\left(\rho+\frac{1}{\rho}\right) & =\frac{1}{2}\left(\rho+p^{2}\right)=-\frac{1}{2} \\
\Theta & =\frac{2 \pi}{3} .
\end{aligned}
$$

Dementsprechend gibt es in der Gruppe $G$ eine Substitution von der dritten Ordnung, welche ein Tripeldreieck invariant lässt.

Auch die Involution conjugierter Durchmesser von $K$ erfährt eine einfache Abbildung, indem sie in die rechtwinklige Strahleninvolution im Mittelpunkte von $\xi^{2}+r_{i}^{2}=1$ verwandelt wird, was 
geometrisch unmittelbar klar ist und analytisch durch die Substitution $\lambda_{2}=i \lambda_{1}$ erkannt wird, welche

$$
\cos \theta=\frac{1}{2}\left(i+\frac{1}{i}\right)=0
$$

ergibt. Es besteht infolgedessen zwischen den Parametern $\varphi_{1}, \varphi_{2}$ geeignet gewählter Endpunkte zweier conjugierter Darchmesser von $K$ die Relation

$$
\varphi_{1}-\varphi_{2}=\frac{\pi}{2}
$$

während die Richtungsconstanten derselben durch die Gleichung

$$
\operatorname{tg} \alpha_{1} \cdot \operatorname{tg} \alpha_{2}=-\frac{b^{2}}{a^{2}}
$$

verknüpft sind, welche, wie dieselbe Betrachtung lehrt, als Verallgemeinerung des Satzes anznseben ist, wonach der Peripheriewinkel über dem Halbkreise ein Rechter ist.

8. Von dem Satze, nach welchem die Höhe eines Tripeldreieckes $r_{1} r_{2} r_{3}$ zugleich Normalen der Ellipse $K \equiv \frac{x^{2}}{a^{2}}+\frac{y^{2}}{b^{2}}-1=0$ sind, kann Gebrauch gemacht werden, um eine einfache Gleichung für die Kiepert'sche Hyperbel irgend eines Dreieckes, welches, wie nachgewiesen wurde, stets als Tripeldreieck einer gewissen Eilipse angeseben werden kann, abzuleiten. Sind nämlich $\alpha, \beta$ die Coordinaten des Höhenschnittpunktes $h$ von $r_{1} r_{2} r_{3}$, so findet man jene vier Punkte von $K$, deren Normalen durch $h$ gehen, indem man $K$ mit dem Kegelschnitte

$$
3-y=\frac{a^{2} y}{b^{2} x}(\alpha-x)
$$

zum Durchschnitte bringt. Da drei dieser Schnittpunkte mit $r_{1}, r_{2}, r_{3}$ identisch sind und der Kegelschuitt

$$
H \equiv b^{2} x(\beta-y)-a^{2} y(\alpha-x)=0
$$

überdies auch $h$ und den Schwerpunkt $(0,0)$ des Dreieckes $r_{1} r_{2} r_{3}$ enthält, so ist er mit der Kiepert'schen Hyperbel dieses Dreieckes identisch.

Um den vierten Schnittpunkt von $K$ und $H$ zu bestimmen, empfiehlt es sich, ein Tripeldreieck, dem ein Scheitel von $K$, etwá $(a, 0)$ angehört, ins Auge zu fassen. Da der Höhenschnittpunkt desselben auf der Abscissenachse liegt, so ist der gesuchte vierte Schnittpunkt in diesem speciellen Falle identisch mit dem Scheitel $(-a, 0)$, welcher jener Stelle diametral gegenüberliegt, in der die 
zu $(a, 0),\left(-\frac{a}{2},+\frac{b \sqrt{3}}{2}\right),\left(-\frac{a}{2},-\frac{b \sqrt{3}}{2}\right)$ gehörigen Krümmungskreise von $K$ den letzteren Kegelschnitt schneiden. Es ist nun zu untersuchen, ob das analoge Verhalten allgemein gilt. Die Entscheidung darüber hängt von der biquadratischen Gleichung $\Phi(\lambda) \equiv\left(\alpha^{2}-b^{2}\right)\left(\lambda^{4}-1\right)+2\left\{(b \beta i-a \alpha) \lambda^{2}+(b \beta i+\alpha \alpha)\right\} \lambda=0$ ab, welche die Parameter der vier Schnittpunkte von $K$ und $H$ liefert and erbalten wird, indem man in der Gleichung von $H$

$$
x=\frac{a}{2} \frac{\lambda^{2}+1}{\lambda}, y=\frac{b}{2 i} \frac{\lambda^{2}-1}{\lambda}
$$

setzt. Ist $\mu$ der Parameter jener Stelle, in welcher die zu $r_{1}, r_{2}, r_{3}$ gehörigen $\mathrm{Krümmungskreise} K$ schneiden, so bestimmt die Gleichung

$$
\lambda^{3} \mu=1
$$

die Parameter des Tripels $r_{1}, r_{2}, r_{3}$ und es ist infolgedessen $\Phi(\lambda)$ durch $\left(\lambda^{3} \mu-1\right)$ theilbar

$$
\Phi(\lambda) \equiv 0 \bmod \left(\lambda^{3} \mu-1\right) .
$$

Es besteuen also unter der Voraussetzung

$$
\Phi(\lambda) \equiv\left(a^{2}-b^{2}\right) \prod_{i=1}^{4}\left(\lambda-\lambda_{i}\right)
$$

die beiden Gleichungen

aus denen

$$
\begin{aligned}
\lambda_{1} \lambda_{2} \lambda_{3} \lambda_{4} & =-1 \\
\lambda_{1} \lambda_{2} \lambda_{3} & =\frac{1}{\mu}
\end{aligned}
$$

$$
i_{4}=-\mu
$$

hervorgeht, ein Parameterwert, welcher dem $\mu$ diametral gegenüberliegenden Punkte von $K$ zukommt.

9. Um den geometrisehen Ort zu finden, welchen die Höhenschnittpunkte $h$ der Tripeldreiecke erzeugen, sollen die Gleichungen der Höhen eines solchen Dreieckes aufgestellt werden. Sie lauten:

$$
\begin{aligned}
(a x-b i y) \lambda_{i}^{3}+\frac{1}{2}\left(a^{2}-b^{2}\right) & =\lambda_{i}\left\{\frac{1}{2}\left(a^{2}-b^{2}\right) \lambda_{i}^{3}+(a x+b i y)\right\} \\
(i & =1,2,3) .
\end{aligned}
$$

Da wegen

$$
\begin{gathered}
\lambda_{2}=p \lambda_{1}, \quad \lambda_{3}=p^{2} \lambda_{1} \\
\lambda_{1}^{3}=\lambda_{2}^{3}=\lambda_{3}^{3}
\end{gathered}
$$


ist, so müssen für den Höbenschnittpunkt $h$ die linke Seite, sowie der Klammerausdruck auf der rechten Seite verschwinden

$$
\begin{aligned}
& (a x-b i y) \lambda_{i}^{3}+\frac{1}{2}\left(a^{2}-b^{2}\right)=0 \\
& \frac{1}{2}\left(a^{2}-b^{2}\right) \lambda_{i}^{3}+(a x+b i y)=0 .
\end{aligned}
$$

Eliminiert man hieraus $\lambda_{i}^{3}$, so ergibt sich für den gesuchten geometrischen Ort die mit $K$ concentrische Ellipse

$$
a^{2} x^{2}+b^{2} y^{2}=\frac{1}{4}\left(a^{2}-b^{2}\right)^{2},
$$

zwischen deren Halbachsen

die Relation

$$
A=\frac{a^{2}-b^{2}}{2 a} \quad B=\frac{a^{2}-b^{2}}{2 b}
$$

besteht.

$$
A a=B b
$$

Auch die Mittelpunkte der den Tripeldreiecken umschriebenen Kreise erfüllen einen Kegelschnitt, welcher mit dem Orte der Höhenschnittpunkte homothetisch ist und die halben Achsenlängen desselben besitzt. Man findet nämlich für die Mittellothe eines Tripeldreieckes:

$$
\begin{gathered}
4(a x-b i y) \lambda_{i}^{3}-\left(a^{2}-b^{2}\right)=\lambda_{i}\left\{4(a x+b i y)-\lambda_{i}^{3}\left(a^{2}-b^{2}\right)\right\} \\
(i=1,2,3)
\end{gathered}
$$

so dass der Mittelpunkt des Umkreises den Gleichungen genügt

$$
\begin{aligned}
4(a x-b i y) \lambda_{i}^{3}-\left(a^{2}-b^{2}\right) & =0 \\
-\left(a^{2}-b^{2}\right) \lambda_{i}^{3}+4(a x+b i y) & =0 .
\end{aligned}
$$

Der fragliche geometrische Ort lautet daher

$$
16\left(a^{2} x^{2}+b^{2} y^{2}\right)=\left(a^{2}-b^{2}\right)^{2},
$$

und seine Halbachsen besitzen die Werte

$$
A^{\prime}=\frac{a^{2}-b^{2}}{4 a}=\frac{A}{2} \quad B^{\prime}=\frac{a^{2}-b^{2}}{4 b}=\frac{B}{2} .
$$

Für $a=b$, d. h. für den Kreis, enthalten die beiden in diesem $\&$ behandelten Orte nur einen reellen Punkt, nämlich den Mittelpunkt $(0,0)$, wie dies sein muss, da für alle einem Kreise eingeschriebenen gleichseitigen Dreiecke die Höhenschnittpunkte mit dieser Stelle coincidieren. 


\section{Die desmische Configuration $\left(\mathbf{2 7}_{9}, \mathbf{8 1}_{3}\right)$.}

1. Auf einem cubischen Kreise vom Geschlechte 1 existiert eine Mannigfaltigkeit von neunelementigen Punktgruppen $\left(r_{1}, \ldots, r_{9}\right)$, welche dadurch charakterisiert ist, dass die zu $r_{1}, \ldots, r_{9}$ gehörigen Krümmungskreise den cubischen Kreis in einem und demselben Punkte $r$ schneiden. Eine Verallgemeinerung $M$ dieser Mannigfaltigkeit wird erhalten, indem man auf irgend einer Curve $C_{3}$ vom Geschlechte 1 zwei Punkte $k, k^{\prime}$ beliebig wählt und denselben die Rolle der absoluten Kreispunkte zuweist, insoferne, als an Stelle der Kreise in der Ebene der circularen $C_{3}$ das lineare System von $\infty^{3}$ Kegelschnitten tritt, welche das Punktepaar $k, k^{\prime}$ enthalten.

Ist $C_{3}$ durch ihre Parameterdarstellung

$$
x: y: z=1: p u: p^{\prime} u
$$

gegeben, - - unter $p u$ ist die von Weierstraf eingeführte doppelperiodische Function verstanden - es ergibt sich für $M$ die Congruenz

$$
3 u \equiv \lambda\left(\operatorname{modd} 2 \omega, 2 \omega^{\prime}\right)
$$

in welcher $\lambda$ einen Parameter bedeutet, bei dessen Variation sämmtliche Gruppen dieser Mannigfaltigkeit gewonnen werden. Die Lösung von $(x)$ lehrt, dass man die Parameter der Elemente einer Gruppe erhält, indem in dem Ausdrucke $\left(u+w_{a b}\right)$ für $w_{a b}$ die sämmtlichen (modd $2 \omega, 2 \omega^{\prime}$ ) incongruenten Periodentripel, also die Parameter der neun $W$ endepunkte

$$
\omega_{a b}=\frac{a .2 \omega+b 2 \omega^{\prime}}{3} \cdot\left(\begin{array}{l}
a=0,1,2 \\
b=0,1,2
\end{array}\right)
$$

substituiert werden.

Aus zwei Gruppen $\left(u+w_{a b}\right),\left(v+w_{a b}\right)$ von $M$ entsteht eine dritte $\left[-(u+v)+w_{a l}\right]$ dadurch, dass man die 81 Graden, von denen jede einen Punkt der ersten Gruppe mit einem solchen der zweiten verbindet. mit der $C_{3}$ zum drittenmale zum Durchschnitte bringt. Von den 81 Schnittpunkten fallen nämlich je 9 zusammen, indem z. B. durch den Punkt $-(u+v)+w_{a b}$, wo $w_{a b}$ den Parameter eines bestimmiten Wendepunktes bezeichnet, sämmtliche Strahlen $\overline{u+w_{A B}, v+w_{A^{\prime} B^{\prime}}}$ gehen, welche der Bedingung

$$
w_{A B}+w_{A^{\prime} B^{\prime}}+w_{a b} \equiv 0
$$

genügen, für welche also die drei Wendepunkte $w_{A B}, w_{A^{\prime} B^{\prime}}, w_{a b}$ auf einer Graden liegen. Durch $w_{a b}$ gehen aber fünf Grade, von 
denen jede drei Wendepunkte enthält, nämlich außer der Wendetangente in $w_{a b}$, welche für $(\beta)$ die Lösung

$$
A \equiv A^{\prime} \equiv a, B \equiv A^{\prime} \equiv b(\bmod 3)
$$

liefert, vier Grade der Wendepunktsconfiguration $\left(9_{4}, 12_{3}\right)$, deren jeder zwei Lösungen von $(\beta)$ correspondieren.

Damit ist nachgewiesen, dass die drei Gruppen $\left(u+w_{a b}\right)$, $\left(v+w_{a b}\right),\left[-(u+v)+w_{a b}\right]$ eine desmische Configuration $\left(27_{9}, 81_{3}\right)$ erzeugen.

Lässt man $v$ unendlich nahe an $u$ heranrücken, so convergiert das abgeleitete System $\left[-(u+v)+w_{a b}\right]$ gegen die Gruppe der Tangentialpunkte $\left(-2 u+w_{a b}\right)$ von $\left(u+w_{a b}\right)$; zugleich redueiert sich die Anzahl der Graden in der desmischen Configuration auf 45 , nämlich auf die neun Tangenten der Punkte $\left(u+w_{a b}\right)$ und die 36 Grade, von denen jede zwei verschiedene Punkte der letzten Gruppe verbindet.

In dem speciellen Falle, wo $u=0$, also $\left(u+w_{a b}\right)$ mit dem Wendepunktssysteme identisch ist, tritt eine weitere Reduction der Graden der Configuration aut 21 ein.

2. Aus der Congruenz $(\alpha)$ ist zu ersehen, dass irgend zwei Punkte $m, m^{\prime}$ der Gruppe $\left(u+w_{a b}\right)$ ein Steiner'sches Punktepaar dritter Ordnung der Curve $C_{3}$ bilden, $d$. h. es lassen sich derselben unendlich viele Sechsecke einschreiben, ron denen drei nicht auf einander folgende Seiten durch $m$ und die drei anderen durch $m^{\prime}$ geben. Unter der gemachten Voraussetzang ist nämlich

$$
m^{\prime} \equiv m+w,
$$

wo $w$ den Parameter eines bestimmten Wendepunktes bezeichnet, was zur Folge hat, dass zwei Grade $m r, m^{\prime} r$ die Curve $C_{3}$, mit der sie den Punkte $r$ gemein haben, noch in zwei weiteren Punkten $n, n^{\prime}$ schneiden, welche wiederum einer Gruppe von $M$ angehören; denn aus

ergibt sich

$$
\begin{aligned}
& m+n+r \equiv 0 \\
& m^{\prime}+n^{\prime}+r \equiv 0
\end{aligned}
$$

$$
n^{\prime} \equiv n+2 w \equiv n-w \text {. }
$$

Es gilt daher das folgende System von Congruenzen:

\%)

$$
\left\{\begin{aligned}
m+n+r & \equiv 0 \\
(m+w)+r+(n+2 w) & \equiv 0 \\
m+(n+2 w)+(r+w) & \equiv 0 \\
(m+w)+(r+w)+(n+w) & \equiv 0 \\
m+(n+w)+(r+2 w) & \equiv 0 \\
(m+w)+(r+2 w)+n & \equiv 0
\end{aligned}\right.
$$


dessen geometrischer Inhalt dahin zu deuten ist, dass thatsächlich ein der $C_{3}$ eingeschriebenes Sechseck $S$ mit den aufeinanderfolgenden Ecken

$$
n, r,(n+2 w),(r+w),(n+w),(r+2 w)
$$

vorliegt, von dem drei nicht an einander stoßende Seiten durch $m$, und die anderen drei durch $(m+w)$ gehen. Von seinen Ecken gehören je drei nicht aufeinanderfolgende einer und derselben Gruppe der Mannigfaltigkeit $M$ an.

Da eine Ecke beliebig gewaiblt werden konnte, so gibt es unendlich viele Sechsecke von der angegebenen Beschaffenheit.

Von einem Steiner'schen $2 n$ Ecke der Curve $C_{3}$ gilt bekanntlich der Satz, dass die Verbindungslinie zweier Ecken, zwischen denen sich eine grade Anzahl anderer befindet, sich ebenfalls um einen festen Punkt der Curve dreht. Wendet man diesen Satz auf das Sechseck $S$ an, indem man je zwei Ecken desselben verbindet, die durch zwei andere getrennt sind, so erhält man

$$
\left\{\begin{array}{l}
(m+2 w)+n+(r+w) \equiv 0 \\
(m+2 w)+(n+w)+r \equiv 0 \\
(m+2 w)+(n+2 w)+(r+2 w) \equiv 0
\end{array}\right.
$$

woraus in Verbindung mit $(\gamma)$ die beiden folgenden Sextupel von Congruenzen fließen:

$$
\left\{\begin{aligned}
m+n+r & \equiv 0 \\
(m+2 w)+r+(n+w) & \equiv 0 \\
m+(n+w)+(r+2 w) & \equiv 0 \\
(m+2 w)+(r+2 w)+(n+2 w) & \equiv 0 \\
m+(n+2 w)+(r+w) & \equiv 0 \\
(m+2 w)+(r+w)+n & \equiv 0 \\
(m+w)+n+(r+2 w) & \equiv 0 \\
(m+2 w)+(r+2 w)+(n+2 w) & \equiv 0 \\
(m+w)+(n+2 w)+r & \equiv 0 \\
(m+2 w)+r+(n+w) & \equiv 0 \\
(m+w)+(n+w)+(r+w) & \equiv 0 \\
(m+2 w)+(r+w)+n & \equiv 0
\end{aligned}\right.
$$

welche übrigens auch unmittelbar hätten aufgestellt werden können. (s) und $(\eta)$ lehren, dass, wenn die Ecken von $S$ in den beiden Reihenfolgen

$$
\begin{aligned}
& n, r, n+w, r+2 w, n+2 w, r+w \\
& n, r+2 w, n+2 w, r, n+w, r+w
\end{aligned}
$$

verbunden werden, zwei weitere Steiner'sche Sechsecke $S^{\prime \prime}$ nnd $S^{\prime \prime}$ entstehen, für welche die zugehörigen Steiner'schen Punktepaare 
dritter Ordnung wit $m$ und $(m+2 w)$, beziehungsweise $(m+w)$ und $(m+2 w)$ identisch sind. Ihre Lagenbeziebung zu $S$ erhellt aus der folgenden schematischen Darstellang, in welcher je drei Seiten, welche mit demselben Punkte des bezüglichen Steiner'schen Punktepaares dritter Ordnung perspectiv liegen, gleich stark dargestellt sind. $\quad \boldsymbol{S}^{\prime}$
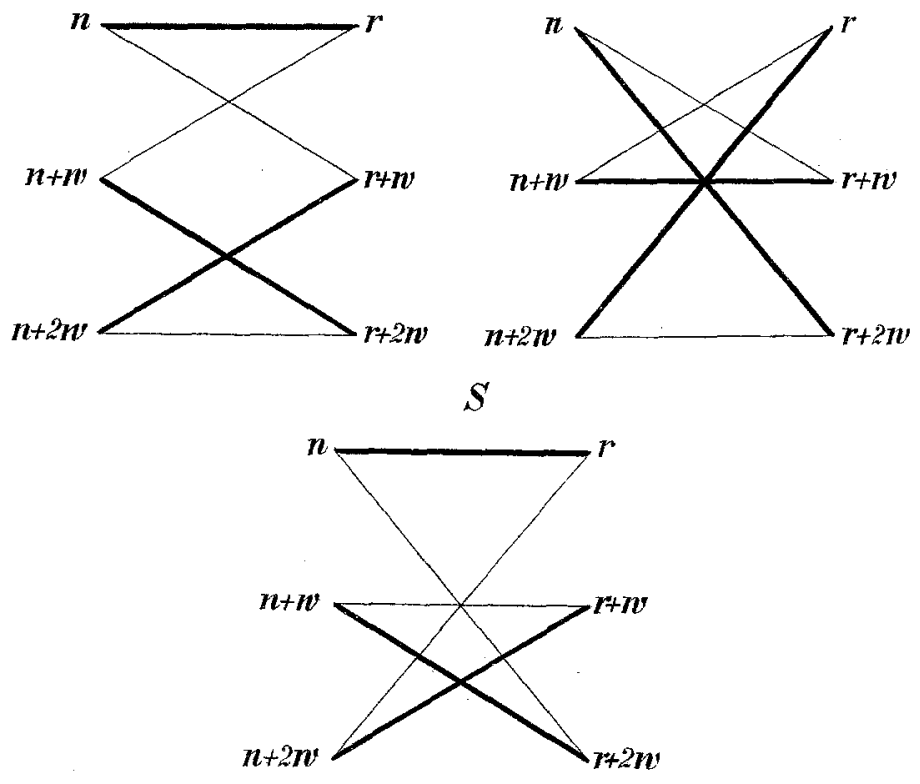

Ein weiteres Resultat ergibt sich aus der Zusammenstellung von (o) mit dem in zwei bestimmte Tripel zerfällten Systeme ( $\gamma$ ).

$$
\begin{aligned}
& \left\{\begin{array}{l}
m+n+r \equiv 0 \\
m+(n+w)+(r+2 w) \equiv 0 \\
m+(n+2 w)+(r+w) \equiv 0
\end{array}\right. \\
& \left\{\begin{array}{l}
(m+w)+n+(r+2 w) \equiv 0 \\
(m+w)+(n+w)+(r+w) \equiv 0 \\
(m+w)+(n+2 w)+r=0
\end{array}\right. \\
& \left\{\begin{array}{l}
(m+2 w)+n+(r+w) \equiv 0 \\
(m+2 w)+(n+w)+v \equiv 0 \\
(m+2 w)+(n+2 w)+(r+2 w) \equiv 0
\end{array}\right.
\end{aligned}
$$

Man erkennt nämlich aus den vorstehenden drei Systemen von Congruenzen, dass von den Dreiecken

$$
(m, m+w, m+2 w),(n, n+w, n+2 w),(r, r+w, r+2 w)
$$


irgend zwei dreifach perspectiv sind, und zwar so, dass die zugehörigen Perspectivitätscentra mit den Ecken des dritten Dreieckes coincidioren, d. h. die angegebenen Dreiecke, deren Ecken je einer Gruppe der Mannigfaltigkeit $M$ angehören, erzeugen eine in der Configuration $\left(27_{9}, 81_{3}\right)$ enthaltene desmische Configuration $\left(9_{3}, 9_{3}\right)$.

3. Um die Gesammtheit der desmischen Configurationen $\left(9_{3}, 9_{3}\right)$ zu überblicken, welche in der Configuration $\left(27_{9}, 81_{3}\right)$ sich vorfinden, ist es nützlich, für die Elemente der Gruppen

$$
\begin{gathered}
G \equiv\left(m+w_{a b}\right), G^{\prime} \equiv\left(n+w_{a b}\right), G^{\prime \prime} \equiv\left(r+w_{a b}\right) \\
r \equiv-(m+n) \\
(a, b) \equiv(00),(01),(02),(10),(11),(12),(20),(21),(22)
\end{gathered}
$$

eine neve Bezeichnung einzuführen. Man setze nämlich

so dass

$$
m+w_{a b} \equiv m_{\alpha b}, n+v_{a b} \equiv n_{a b}, r+w_{a b} \equiv r_{a b},
$$

$$
\begin{gathered}
m \equiv m_{00}, n \equiv n_{00}, r \equiv r_{00} \\
G \equiv\left(m_{a b}\right), G^{\prime} \equiv\left(n_{\alpha b}\right), G^{\prime \prime} \equiv\left(r_{a b}\right)
\end{gathered}
$$

wird. Alsdann liegen wegen

$$
m_{00}+n_{00}+r_{00} \equiv 0
$$

die drei Punkte $m_{a b}, n_{a^{\prime} b^{\prime}}, r_{a^{\prime \prime} b^{\prime \prime}}$ dann und nur dann auf einer Graden, $\mathrm{d}$. h. es ist

$$
m_{\alpha b}+n_{a^{\prime} b^{\prime}}+r_{a^{\prime \prime} b^{\prime \prime}} \equiv 0,
$$

wenn die beiden Congruenzen

$$
\begin{aligned}
& a+a^{\prime}+a^{\prime \prime} \equiv 0 \\
& b+b^{\prime}+b^{\prime \prime} \equiv 0
\end{aligned} \quad \bmod 3
$$

erfüllt sind. Dieselben können auch durch das äquivalente System

$$
\begin{aligned}
& a-a^{\prime \prime} \equiv a^{\prime \prime}-a^{\prime} \equiv a^{\prime}-a \\
& b-b^{\prime \prime} \equiv b^{\prime \prime}-b^{\prime} \equiv b^{\prime}-b \quad \bmod 3
\end{aligned}
$$

ersetzt werden, woraus hervorgeht, dass in $(x)$ entweder alle drei Indicespaare $(a b),\left(a^{\prime} b^{\prime}\right),\left(a^{\prime \prime} b^{\prime \prime}\right)$ verschieden, oder alle drei gleich sind, da aus

die Congruenzen

$$
a^{\prime} \equiv a, b^{\prime} \equiv b
$$

$$
a^{\prime \prime} \equiv a, b^{\prime \prime} \equiv b
$$

folgen.

In dem Falle, wo die drei Indicespaare verschieden sind, bleibt, wie aus ( $x$ ) ersichtlich ist, die Congruenz

$$
m_{\alpha b}+n_{a^{\prime} b^{\prime}}+r_{a^{\prime \prime} b^{\prime \prime}} \equiv 0
$$

aufrecht, wenn in ihr die Indicessysteme auf alle möglichen Arten vertauscht werden; es enthält demnach jeder Term in der Entwicklung der Determinante 


$$
\left|\begin{array}{lll}
m_{a b} & n_{a b} & r_{a b} \\
m_{a^{\prime} b^{\prime}} & n_{a^{\prime} b^{\prime}} & r_{a^{\prime} b^{\prime}} \\
m_{a^{\prime \prime} b^{\prime \prime}} & n_{a^{\prime \prime} b^{\prime \prime}} & r_{a^{\prime \prime} b^{\prime \prime}}
\end{array}\right|
$$

drei Punkte auf einer Graden; eine Eigenthümlichkeit, welche auch den Elementen einer jeden Horizontalreihe zukommt. Es ergeben sich auf diese Weise neun Grade, die auf eine und nur eine Art in drei Tripel zerlegt werden können, von denen jedes neun verschiedene Punkte der Configuration $\left(27_{9}, 81_{3}\right)$ enthält:

$$
\begin{array}{r}
\left\{\begin{array}{l}
m_{a b}+n_{a b}+r_{a b} \equiv 0 \\
m_{a^{\prime} b^{\prime}}+n_{a^{\prime} b^{\prime}}+r_{a^{\prime} b^{\prime}} \equiv 0 \\
m_{a^{\prime \prime} b^{\prime \prime}}+n_{a^{\prime \prime} b^{\prime \prime}}+r_{a^{\prime \prime} b^{\prime \prime}} \equiv 0
\end{array}\right. \\
\left\{\begin{array}{l}
m_{a b}+n_{a^{\prime} b^{\prime}}+r_{a^{\prime \prime} b^{\prime \prime}} \equiv 0 \\
m_{a^{\prime} b^{\prime}}+n_{a^{\prime \prime} b^{\prime \prime}}+r_{a b} \equiv 0 \\
m_{a^{\prime \prime} b^{\prime \prime}}+n_{a b}+r_{a^{\prime} b^{\prime}} \equiv 0
\end{array}\right. \\
\left\{\begin{array}{l}
m_{a b}+n_{a^{\prime \prime} b^{\prime \prime}}+r_{a^{\prime} b^{\prime}} \equiv 0 \\
m_{a^{\prime} b^{\prime}}+n_{a b b}+r_{a^{\prime \prime} b^{\prime \prime}} \equiv 0 \\
m_{a^{\prime \prime} b^{\prime \prime}}+n_{a^{\prime} b^{\prime}}+r_{a b} \equiv 0
\end{array}\right.
\end{array}
$$

Aus $(\mu),\left(\mu^{\prime}\right),\left(\mu^{\prime \prime}\right)$ geht hervor, dass die Dreiecke

$$
\left(m_{\alpha b}, m_{a^{\prime} b^{\prime}}, m_{a^{\prime \prime} b^{\prime \prime}}\right),\left(n_{a b}, n_{a^{\prime} b^{\prime}}, n_{a^{\prime \prime} b^{\prime \prime}}\right),\left(r_{a b}, r_{a^{\prime} b^{\prime}}, r_{a^{\prime \prime} b^{\prime \prime}}\right)
$$

eine desmische Configuration $\left(9_{3} .9_{3}\right)$ erzeugen. Indessen ist dieselbe von der im vorigen $\S$ nachgewiesenen nicht verschieden. Es gelten närnlich die Congruenzen

$$
\begin{array}{ll}
m_{a^{\prime} b^{\prime}} \equiv m_{a b}+w_{a^{\prime}-a, b^{\prime}-b} & m_{a^{\prime \prime} b^{\prime \prime}} \equiv m_{a b}+w_{a^{\prime \prime}-a, b^{\prime \prime}-b} \\
n_{a^{\prime} b^{\prime}} \equiv n_{a b}+w_{a^{\prime}-a, b^{\prime}-b} & n_{a^{\prime \prime} b^{\prime \prime}} \equiv n_{a b}+w_{a^{\prime \prime}-a, b^{\prime \prime}-b} \\
r_{a^{\prime} b^{\prime}} \equiv r_{a b}+w_{a^{\prime}-a, b^{\prime}-b} & r_{a^{\prime \prime} b^{\prime \prime}} \equiv r_{a b}+w_{a^{\prime \prime}-a, b^{\prime \prime}-b}
\end{array}
$$

worin wegen $(\lambda)$

$$
w_{a^{\prime \prime}-a, b^{\prime \prime}-b} \equiv w_{-\left(a^{\prime}-a\right),-\left(b^{\prime}-b\right)} \equiv-w_{a^{\prime}-a, b^{\prime}-b}=2 w_{a^{\prime}-a, b^{\prime}-b^{\prime}} \text {. }
$$

Es sind daher in der That die angegebenen Triangel in der Form darstelbar :

$$
\begin{aligned}
& \left(m_{\alpha b}, m_{a b}+w_{a^{\prime}-a, b^{\prime}-b}, m_{a b}+2 w_{a^{\prime}-a, b^{\prime}-b}\right) \\
& \left(n_{\alpha b}, n_{a b}+w_{a^{\prime}-a, b^{\prime}-b}, n_{\alpha b}+2 w_{a^{\prime}-a, b^{\prime}-b}\right) \\
& \left(r_{a b}, r_{a b}+w_{a^{\prime}-a, b^{\prime}-b}, r_{a b}+2 w_{a^{\prime}-a, b^{\prime}-b}\right) .
\end{aligned}
$$

4. Das Tripel $(\mu)$ ist ein Bestandtheil der neunelementigen Strahlengruppe

$$
\left[\begin{array}{l}
m_{\alpha \beta}+n_{\alpha \beta}+r_{\alpha \beta} \equiv 0 \\
(\alpha, \beta) \equiv(00),(01),(02),(10),(11),(12),(20),(21),(22)
\end{array}\right]
$$


welche durch die Eigenschaft ausgezeichnet ist, alle 27 Punkte der Configuration $\left(27_{3}, 81_{3}\right)$ zu enthalten. Jedes derartige System von neun Strahlen dieser Configuration, von denen also keine zwei sich auf der Curve $C_{3}$ schneiden, soll als ein vollständiges System bezeichnet werden.

Wie eine einfache Betrachtung lebrt, stellt

$$
\begin{aligned}
N_{a b} & \equiv\left[\begin{array}{l}
\left(m_{\alpha \beta}+2 w_{\alpha b}\right)+\left(n_{\alpha \beta}+w_{\alpha, b}\right)+r_{\alpha \beta} \equiv 0 \\
(\alpha \beta) \equiv(00),(01), \ldots(22)
\end{array}\right] \\
& \equiv\left[\begin{array}{l}
m_{\alpha+2 \alpha, \beta+2 b}+n_{\alpha+a, \beta+b}+r_{\alpha \beta} \equiv 0 \\
(\alpha \beta) \equiv(00),(01) \ldots(22)
\end{array}\right]
\end{aligned}
$$

wenn unter $w_{a b}$ irgend ein bestimmter Wendepunkt verstanden wird, eir. vollständiges System dar. Den neun verschiedenen Wendepunkten entsprechend existieren demnach neun vollständige Systeme von der Form ( $\rho$ ), unter denen, dem Wendepunkte $w_{a b} \equiv 0$ correspondierend, die Gruppe (v) vorkommt.

Da ein jeder Strahl der Configuration $\left(27_{9}, 81_{3}\right)$ in der Form

$$
\left(m_{a \beta}+2 w_{a b}\right)+\left(n_{\alpha \beta}+w_{a b}\right)+r_{\alpha \beta} \equiv 0
$$

darstellbar ist, so erschöpfen die neun vollständigen Systeme $N_{00}$, $N_{01} \ldots N_{22}$ sämmtliche 81 Grade dieser Contiguration, woraus zugleich hervorgeht, dass keine zwei derselben einen gemeinschaftlichen Strabl enthalten.

Durch jedes der Systeme $N_{a z}$ wird eine Correspondenz zwischen den Elementen von $G, G^{\prime}, G^{\prime \prime}$ erzeugt, in welcher drei solche Punkte dieser Gruppen einander homolog sind, die einem und demselben Strahle von $N_{a b}$ angehören. In der zwischen $G$ and $G^{\prime}$ bestehenden Verwandtschaft entsprechen sich also $\left(m_{\alpha \beta}+2 w_{a b}\right)$ und $\left(n_{a \beta}+w_{a b}\right)$ für $(\alpha \beta)=(00),(01), \ldots(22)$. Nun ist es von Wichtigkeit, dass diese Verwandtschaft auch rein geometrisch definiert werden kann. Verschiebt man nämlich den Punkt $\left(m_{00}+2 w_{a b}\right)$ stetig längs der Curve $C_{3}$, bis er nach $\left(n_{00}+w_{a b}\right)$ gelangt und lässt gleichzeitig auch die übrigen Elemente von $G$ auf der Curve sich fortbewegen, und zwar so, dass die bewegten Punkte in jedem Augenblicke eine Gruppe der Mannigfaltigkeit $M$ repräsentieren, so wird am Schlusse der Bewegung wegen

$$
\begin{gathered}
m_{\alpha \beta}+2 w_{\alpha b} \equiv\left(m_{00}+2 w_{a b}\right)+w_{a \beta} \\
n_{\alpha \beta}+w_{\alpha b} \equiv\left(n_{00}+w_{a b}\right)+w_{\alpha \beta}
\end{gathered}
$$

$\left(m_{\alpha \beta}+2 w_{a b}\right)$ für jedes der neun Indexsysteme $(\alpha \beta)$ in $\left(n_{\alpha \beta}+w_{\alpha b}\right)$ iibergeführt. Damit ist die geometrische Bedeutung der neun vollständigen Systeme $N_{a b}$, von denen jedes durch Angabe eines seiner Strahlen unzweideutig bestimmt ist, erkannt. Wegen der ausgezeichneten Stellung, welche sie innerhalb der Gesammtheit der vollständigen Systeme einnehmen, sollen dieselben als "normale" vollständige Systeme oder kurz als "Normalsysteme" bezeichnet werden. 
Ihre Gleichberechtigung unter einander ergibt sich auch auf analytischem Wege mittelst der Substitution

$$
m_{00}+2 w_{a b} \equiv m_{00}^{\prime}, n_{00}+w_{0 b} \equiv n_{002}^{\prime} r_{00} \equiv r_{0}^{\prime} \text {, }
$$

durch welche für $N_{a b}$ die mit (v) übereinstimmende Darstellung

$$
N_{a b} \equiv\left[\begin{array}{l}
m_{\alpha \beta \beta}^{\prime}+n_{\alpha \beta \beta}^{\prime}+r_{\alpha \beta}^{\prime} \equiv 0 \\
(\alpha, \beta) \equiv(00) \ldots(22)
\end{array}\right]
$$

erhalten wird. Dieselbe besagt, dass es möglich ist, einem jeden Normalsysteme ein beliebiges der neun Indexpare zuzuordnen, also z. B. $N_{a b}^{\prime} \equiv N_{00}^{\prime}$ zu setzen.

Dieselbe Betrachtung liefert auch die Bedingung dafür, dass die zwei Graden

$$
\begin{aligned}
& m_{a b}+n_{a^{\prime} b^{\prime}}+r_{a^{\prime \prime} b^{\prime \prime}} \equiv 0 \\
& m_{\alpha \beta}+n_{\alpha^{\prime} \beta^{\prime}}+r_{a^{\prime \prime} \beta^{\prime \prime}} \equiv 0
\end{aligned}
$$

einem und demselben Normalsysteme angehören. Aus dem Congruenzsysteme

$$
m_{a b}-m_{a \beta} \equiv n_{a^{\prime} b^{\prime}}-n_{\alpha^{\prime} \beta^{\prime}} \equiv r_{a^{\prime \prime} b^{\prime \prime}}-r_{\alpha^{\prime \prime} \beta^{\prime \prime}} ;
$$

welches mit

$$
w_{a-a, b-\beta} \equiv w_{a^{\prime}-a^{\prime}, b^{\prime}-\beta^{\prime}} \equiv w_{a^{\prime \prime}-a^{\prime \prime}, b^{\prime \prime}-\beta^{\prime \prime}}
$$

identisch ist, folgt nämlich für das gesuchte Criterium:

$$
\begin{aligned}
& a-\alpha \equiv a^{\prime}-\alpha^{\prime} \equiv a^{\prime \prime}-a^{\prime \prime} \\
& b-\beta \equiv b^{\prime}-\beta^{\prime} \equiv b^{\prime \prime}-\beta^{\prime \prime} . \quad \bmod 3
\end{aligned}
$$

Als eine Anwendung von $(\sigma)$ findet man auf Grund von $(\lambda)$, dass die beiden Strahlentripel $\left(\mu^{\prime}\right)$ and $\left(\mu^{\prime \prime}\right)$ des $\$$. 3 die Eigenschaft von ( $\mu$ ) theilen, einem Normalsysteme anzugehören. Man überzeugt sich leicht, dass dieselben in $N_{a}^{\prime \prime}-a, b^{\prime \prime}-b$, , beziehungsweise $N_{a-a^{\prime \prime}, b-b^{\prime \prime}}$ enthalten sind.

5 . Das dem Normalsysteme $N_{00}$ angehörige Trippel $(\mu)$ des $\$ .3$ hatte den Bedingungen zu genügen, dass die Indexsysteme $(a b),\left(a^{\prime} b^{\prime}\right),\left(a^{\prime \prime} b^{\prime \prime}\right)$ von einander verschieden sind, und übrrdies die Congruenzen $(x)$ erfüllt werden. $\mathrm{Da}$ bei einer willkürlichen Wabl von $(a, b)$ für $\left(a^{\prime}, b^{\prime}\right)$ acht Wertsysteme zulässig sind, so erkennt man mit Rücksicht auf den symmetrischen Bau von $(x)$, dass es in $N_{00} \frac{9.8}{6}=12$ Tripel von der Form $(\mu)$ gibt, deren zugehörige Indexsysteme durch die Horizontalreihen, die Verticalreihen und die Glieder der Entwicklung der Determinante

$$
-\left|\begin{array}{lll}
(00) & (01) & (02) \\
(10) & (11) & (12) \\
(20) & (21) & (22)
\end{array}\right|
$$


geliefert werden. Nun ist jedem dieser Tripel eine desmische Configuration $\left(9_{3}, 9_{3}\right)$ zugeordnet, weshalb sich bei Benutzung särmmtlicher neun Normalsysteme 12.9 derartige Configurationen ergeben, von denen jedoch jede dreimal gezählt ist, da je drei der in Betracht kommenden Tripel, wie $(\mu),\left(\mu^{\prime}\right),\left(\mu^{\prime \prime}\right)$ eine und dieselbe Configuration erzeugen. Im ganzen erhält man daher auf dem angegebenen Wege 36 in $\left(27_{9}, 81_{3}\right)$ enthaltene desmische Configurationen $\left(9_{3}, 9_{3}\right)$, und es ist nicht schwer nachzuweisen, dass es anßer denselben keine weiteren gibt. Wenn nämlich die Dreiecke

$$
\left(m_{a b}, m_{a^{\prime} b^{\prime}}, m_{a^{\prime \prime} b^{\prime \prime}}\right)\left(n_{c d}, n_{c^{\prime} d^{\prime}}, n_{a^{\prime \prime} d^{\prime \prime}}\right)\left(r_{e f}, r_{\epsilon^{\prime} f^{\prime}}, r_{e^{\prime \prime} f^{\prime \prime}}\right)
$$

zu einer desmischen Configuration Anlass geben, so besteht, wenn die Bezeichnung passend gewählt wird, das folgende System von. Congruenzen :

$$
\begin{array}{r}
\left\{\begin{array}{l}
m_{a b}+n_{c d}+r_{e f} \equiv 0 \\
m_{a^{\prime} b^{\prime}}+n_{c^{\prime} d^{\prime}}+r_{e^{\prime} f^{\prime}} \equiv 0 \\
m_{a^{\prime \prime} b^{\prime \prime}}+n_{a^{\prime \prime} d^{\prime \prime}}+r_{e^{\prime \prime} f^{\prime \prime}} \equiv 0
\end{array}\right. \\
\left\{\begin{array}{l}
m_{a b}+n_{c^{\prime} d^{\prime}}+r_{e^{\prime \prime} f^{\prime \prime}} \equiv 0 \\
m_{a^{\prime} b^{\prime}}+n_{c^{\prime \prime} d^{\prime \prime}}+r_{e f} \equiv 0 \\
m_{a^{\prime \prime} b^{\prime \prime}}+n_{c d}+r_{e^{\prime} f^{\prime}} \equiv 0
\end{array}\right. \\
\left\{\begin{array}{l}
m_{a b}+n_{c^{\prime \prime} d^{\prime \prime}}+r_{e^{\prime} f^{\prime}} \equiv 0 \\
m_{a^{\prime} b^{\prime}}+n_{c d}+r_{e^{\prime \prime} f^{\prime \prime}} \equiv 0 \\
m_{a^{\prime \prime} b^{\prime \prime}}+n_{c^{\prime} d^{\prime}}+r_{e f} \equiv 0
\end{array}\right.
\end{array}
$$

Aus den beiden Congruenzen in $\left(\tau^{\prime}\right)$ und $\left(\tau^{\prime \prime}\right)$, welche als gemeinsamen dritten Posten $r_{e^{\prime \prime}}^{\prime \prime} f^{\prime \prime}$ aufweisen, folgt:

also

$$
\begin{array}{ll}
a+c^{\prime}+e^{\prime \prime} \equiv 0 & b+d^{\prime}+f^{\prime \prime} \equiv 0 \\
a^{\prime}+c+e^{\prime \prime} \equiv 0 & b^{\prime}+d+f^{\prime \prime} \equiv 0
\end{array} \quad \bmod 3,
$$

$$
a-a^{\prime} \equiv c-c^{\prime} \quad b-b^{\prime} \equiv d-d^{\prime}
$$

Mittelst analoger Betrachtungen findet man zwischen den neun Indexpaaren $(a b), \ldots\left(e^{\prime \prime} f^{\prime \prime}\right)$ weitere ähnliche Beziehungen, welche zufolge $(c)$ aussagen, dass $(c),\left(\tau^{\prime}\right),\left(\tau^{\prime \prime}\right)$ je in einem Normalsysteme enthalten sind, und in der gleichen Beziehnung zu einander stehen, wie $(\mu),\left(\mu^{\prime}\right),\left(\mu^{\prime \prime}\right) ;$ q. e. d.

6. Die Normalsysteme ermöglichen es, die Gesammtheit der vollständigen Systeme in einer gewissen Hinsicht zu classificieren.

Irgend ein vollständiges System $S$ hat nämlich mit jedem der Normalsysteme $N_{a b}$ eine gewisse Anzahl $\alpha_{a b}$ von Strahlen gemeinsam, deren Complex

$$
\left(\alpha_{00}, \alpha_{01}, \alpha_{02}, \alpha_{10}, \alpha_{11}, \alpha_{12}, \alpha_{20}, \alpha_{31}, \alpha_{22}\right)
$$

als eine Charakteristik von $S$ angesehen werden kann. Zufolge 
der Definition ist

$$
\Sigma \alpha_{a b}=9
$$

Wenn von den Normalsystemen abgesehen wird, für welche je eines der $\alpha$ den Wert 9 besitzt, während die übrigen verschwinden, so erreichen die Zahlen a höchstens den Wert 6 , da zwei vollständige Systeme offenbar identisch sind, wenn sie 7 gemeinschaftliche Strahlen enthalten:

$$
\alpha_{a b}==7,8 \text {. }
$$

Was nun die vollständigen Systeme $\Sigma$ anlangt, welche mit einem Normalsysteme ein Sextupel $s$ gemein haben, so ist aus dem Vorhergehenden klar, dass dieselben den gleichen Bau aufweisen wie die beiden vollständigen Systeme $\left[\left(N_{00}-\mu\right)+\mu^{\prime}\right]$ und $\left[\left(N_{00}-\mu\right)+\mu^{\prime \prime}\right]$, welche das Sextupel $\left(N_{00}-\mu\right)$ von $N_{00}$ enthalten; $d h$. das Tripel $(\Sigma-s)$ ist ebenfalls in einem Normalsysteme enthalten, und gibt Anlass zur Exzeugung einer gewissen desmischen Configuration $\left(9_{3}, 9_{3}\right)$. In der Charakteristik eines vollständigen Systems $\Sigma$ verschwinden daher 7 der Zahlen $\alpha$, während die beiden übrigen die Werte 6 und 3 besitzen.

Ferner ist klar, dass die Anzahl der Systeme $\mathbf{2}$, welche mit einem bestimmten Normalsysteme, z. B. $N_{00}$, ein Sextupel gemein haben, doppelt so groß ist als die Anzahl der in diesem Normalsysteme vorkommenden Tripel von der Form $(u)$. Dieselbe besitzt demnach den Wert 24, aus welchem durch Multiplication mit 9 hervorgeht, dass die Systeme $\Sigma$ in der Anzahl 216 vertreten sind.

Von den 24 Systemen $\Sigma$, welche je ein Sextupel eines und desselben Normalsystems $N_{a b}$ enthalten, sind je drei, $\Sigma_{1} \equiv s_{1}+t_{1}$, $\Sigma_{2} \equiv s_{2}+t_{2}, \Sigma_{3} \equiv s_{3}+t_{3}$, von der Beschaffenheit, dass sie mit einem und demselben zweiten Normalsysteme $N_{\alpha^{\prime} b^{\prime}}$ je ein Tripel $t_{1}, t_{2}, t_{3}$ gemein haben. Alsdann sind die Sextupel $s_{1}, s_{2}, s_{3}$ paarweise verschieden, da zwei vollständige Systeme mit gemeinschaftlichem Sextupel, wie $\left[\left(N_{00}-\mu\right)+\mu^{\prime}\right]$ und $\left[\left(N_{00}-\mu\right)+\mu^{\prime \prime}\right]$ anßerdem noch Tripel enthalten, welche nach dem früher Bewiesenen verschiedenen Normalsystemen angehören. Daraus folgt, dass $t_{1}, t_{2}, t_{3}$ lauter verschiedene Strablen enthalten. Denn fände sich z. B. der Strahl

$$
m_{\alpha \beta}+n_{\alpha^{\prime} \beta^{\prime}}+r_{\alpha^{\prime \prime} \beta^{\prime \prime}} \equiv 0
$$

sowohl in $t_{1}$, als auch in $t_{2}$ vor, so könnte keine von den drei Graden in $N_{a b}$, welche beziehungsweise durch $m_{a \beta}, n_{\alpha^{\prime} \beta^{\prime}}, r_{a^{\prime \prime} \beta^{\prime \prime}}$ hindurchgehen, in $s_{1}$ oder $s_{2}$ virkommen; dann wäre aber nothwendig $s_{1} \equiv s_{2}$; was unmöglich ist. Es erschöpfen daher die Tripel $t_{1}, t_{2}, t_{3}$ thatsächlich das Normalsystem $N_{a^{\prime} b^{\prime}}^{\prime}$ :

$$
N_{a^{\prime} b^{\prime}} \equiv t_{1}+t_{2}+t_{3} \text {. }
$$

